\title{
Susceptibility of human Plasmodium knowlesi infections to anti-malarials
}

\author{
Farrah A Fatih ${ }^{1}$, Henry M Staines ${ }^{1}$, Angela Siner ${ }^{2}$, Mohammed Atique Ahmed ${ }^{2}$, Lu Chan Woon ${ }^{3}$, Erica M Pasini ${ }^{4}$, \\ Clemens HM Kocken ${ }^{4}$, Balbir Singh ${ }^{2}$, Janet Cox-Singh ${ }^{2,5}$ and Sanjeev Krishna ${ }^{1,2^{*}}$
}

\begin{abstract}
Background: Evidence suggests that Plasmodium knowlesi malaria in Sarawak, Malaysian Borneo remains zoonotic, meaning anti-malarial drug resistance is unlikely to have developed in the absence of drug selection pressure. Therefore, adequate response to available anti-malarial treatments is assumed.

Methods: Here the ex vivo sensitivity of human P. knowlesi isolates in Malaysian Borneo were studied, using a WHO schizont maturation assay modified to accommodate the quotidian life cycle of this parasite. The in vitro sensitivities of $P$. knowlesi $\mathrm{H}$ strain adapted from a primate infection to in vitro culture (by measuring the production of Plasmodium lactate dehydrogenase) were also examined together with some assays using Plasmodium falciparum and Plasmodium vivax.

Results: Plasmodium knowlesi is uniformly highly sensitive to artemisinins, variably and moderately sensitive to chloroquine, and less sensitive to mefloquine.

Conclusions: Taken together with reports of clinical failures when $P$. knowlesi is treated with mefloquine, the data suggest that caution is required if using mefloquine in prevention or treatment of $P$. knowlesi infections, until further studies are undertaken.
\end{abstract}

Keywords: Artemisinin, Artemether, Artesunate, Dihydroartemisinin, DHA, Chloroquine, Mefloquine, Malaria

\section{Background}

From its natural simian hosts in Southeast Asia, Plasmodium knowlesi has emerged as a significant human pathogen, particularly in Malaysian Borneo [1-3]. Human $P$. knowlesi infections cause febrile illnesses that can rapidly progress to severe and sometimes fatal outcomes [4]. Ominously, the incidence of $P$. knowlesi malaria is increasing in geographic areas where Plasmodium falciparum and Plasmodium vivax are coming under control, thereby threatening the aim of eliminating malaria [5]. Determining the efficacy of conventional anti-malarials against P. knowlesi is a priority, particularly as there are no reports of anti-malarials assessed against human isolates of P. knowlesi ex vivo.

\footnotetext{
* Correspondence: s.krishna@sgul.ac.uk

'Division of Clinical Sciences, Centre for Infection and Immunity, St. George's, University of London, London SW17 ORE, UK

${ }^{2}$ Malaria Research Centre, University Malaysia Sarawak, Kuching 93150, Malaysia
}

Full list of author information is available at the end of the article
Here, the drug sensitivity profiles of $P$. knowlesi isolates obtained from patients being recruited into a study of the pathophysiology of knowlesi malaria in an endemic area of Sarawak, Malaysian Borneo were investigated. Currently, the WHO recommends artemisinin-based combination therapy (ACT) as first-line treatment for malaria in most endemic areas, so artemisinin and its clinically useful derivatives artesunate, dihydroartemisinin (DHA) and artemether were tested. Mefloquine, used as a partner drug in certain artemisinin-based combinations and in prophylaxis against malaria, and chloroquine that is recommended for treatment of Plasmodium malariae (the species which $P$. knowlesi is often confused with when diagnosed by microscopy) were also included. In addition, results from $P$. falciparum and $P$. vivax studied contemporaneously are presented, together with those obtained with the $\mathrm{H}$ strain laboratory isolate of $P$. knowlesi (cultured in vitro in rhesus erythrocytes) to confirm the methodologies used. Insights into the drug susceptibility patterns of this important emerging parasite, may prove useful in guiding 
the best choice of anti-malarial treatment regimens for P. knowlesi infection.

\section{Methods}

\section{Patient recruitment}

Plasmodium isolates were obtained from patients presenting to hospitals in Sarikei and Sibu. Informed written consent was obtained from all patients entered into this study, which was approved by the Malaysian Ministry of Health's Medical Research and Ethics Committee, and the Sarawak State Planning Unit. Infecting species was confirmed by Plasmodium species-specific nested-PCR assays [6] and only patients with single species infections were retained in the study.

\section{Blood collection and ex vivo parasite development in growth assays}

Pre-treatment venous blood from each patient was collected into EDTA. Parasitaemia and the asexual stage of development were determined by Giemsa-stained thin film microscopy. Whole blood ( 2.5 ml) was washed twice without centrifugation to avoid haemolysis, before resuspending in RPMI 1640 complete medium supplemented with $20 \mathrm{mM}$ D-glucose, $40 \mathrm{mM}$ HEPES, $25 \mathrm{mg} / \mathrm{l}$ gentamicin sulphate, and $15 \% v / v$ human $\mathrm{AB}$ plasma with $0.2 \mathrm{mM}$ hypoxanthine.

Growth inhibition by anti-malarials was assessed by quantifying schizont maturation using an adapted WHO Mark III assay [7]. Species of Plasmodium tested and the time lag in maturation seen previously ex vivo were allowed for in these assays [8]. While more complex counting procedures have been used to study $P$. vivax parasites that, like $P$. knowlesi, have mature parasites present in circulating blood [9], only the timing of assays was altered. This is because the $P$. knowlesi isolates contained predominantly immature parasites (Table 1) and results from a parallel study on tightly synchronised immature laboratory $P$. knowlesi $\mathrm{H}$ strain parasites with artesunate using the $p \mathrm{LDH}$ (see below) and Mark III assays were comparable with each other and with the data derived from isolates (see Results).

Aliquots $(100 \mu \mathrm{l})$ of no drug control and serial dilutions of the anti-malarial compounds in culture medium (final concentrations of 0.25 to $25 \mathrm{nM}$ for artemisinin and derivatives and $1.25 \mathrm{nM}$ to $1 \mu \mathrm{M}$ for chloroquine and mefloquine) were dispensed into 96-well plates, and parasites were added (100 $\mu \mathrm{l}$ at $2 \%$ haematocrit). Incubation was in $5 \% \mathrm{O}_{2}, 5 \% \mathrm{CO}_{2}, 90 \% \mathrm{~N}_{2}$ at $37^{\circ} \mathrm{C}$ until the majority of parasites reached the schizont stage (with at least 3 nuclei after $12-17 \mathrm{~h}$ for $P$. knowlesi). Monitoring of maturation was undertaken every $2 \mathrm{~h}$ by examination of fixed, Giemsa stained thin blood films, taken from a parallel culture to those of the drug exposed cultures. When at least half of the parasites in the monitoring culture had reached the schizont stage of development, the drug exposed cultures and their controls were harvested, as thick films on glass slides, and examined. The duration of in vitro development for each isolate (as determined above), which also equates to the time of drug exposure, is given in Table 1. Thick films were fixed by air drying for at least $24 \mathrm{~h}$, stained with Giemsa, and mounted to protect slides during transportation.

As thick films are easily damaged, five replicates were prepared for each drug concentration and 3 replicates then counted for each experimental condition. Counting was in a blinded fashion to avoid bias. Thick films were counted according to the WHO Mark III protocol. At least 200 asexual parasites were counted. Fields of view were consecutive, starting at the left edge of each blood film and moving stepwise in a uniform direction (to ensure no overlap). Asexual parasites were grouped into either trophozoites or schizonts (defined as asexual parasites displaying 1-2 nuclei and 3 or more nuclei, respectively).

Table 1 Patient isolate data

\begin{tabular}{llllll}
\hline Isolate & Species & \% starting parasitaemia & \% schizont at start of assay & Duration of in vitro development (h) & \% schizont at end of assay \\
\hline P0002 & $P k$ & 0.4 & 15 & 12 & 80 \\
P0003 & $P k$ & 0.6 & 10 & 12 & 86 \\
P0006 & $P k$ & 0.8 & 33 & 17 & 53 \\
P0009 & $P k$ & $7.0^{a}$ & 17 & 15 & 94 \\
P0010 & $P k$ & 0.8 & 6 & 12 & 63 \\
P0011 & $P k$ & 1.3 & 8 & 14 & 54 \\
P0007 & $P f$ & $2.0^{a}$ & 0 & 31 & 50 \\
P0001 & $P V$ & 0.5 & 20 & 18 & 58 \\
P0013 & $P V$ & 0.5 & 30 & 29 & 60
\end{tabular}

${ }^{a}$ Above Mark III assay cut-off (for examination of $P$. falciparum mono-infections), assuming a parasitaemia of $1.6 \%$ equates to approximately 80,000 parasites per $\mu \mathrm{I}$ of blood, and was used undiluted. Note that in both cases good schizont development was observed.

For each isolate, the species, parasitaemia and change in development over time are presented. 


\section{Growth assay measuring Plasmodium lactate dehydrogenase ( $p$ LDH)}

Rhesus monkey red blood cells for in vitro P. knowlesi culture were obtained under protocols approved by the independent institutional ethical committee (DEC) according to Dutch and European laws.

The efficacy of the anti-malarial compounds was also assessed in vitro against the laboratory maintained P. knowlesi $\mathrm{H}$ strain $[10,11]$, by measuring the production of $p \mathrm{LDH}$ [12]. $p \mathrm{LDH}$ catalyzes the conversion of 3-acetylpyridine adenine dinucleotide (APAD) and lactate to APADH and pyruvate. The enzyme diaphorase subsequently converts nitro blue tetrazolium (NBT) to nitro blue formazan $(\mathrm{NBF})$, using APADH as a reducing agent. NBF can be measured at a wavelength of $655 \mathrm{~nm}$. Serial dilutions of the anti-malarial compounds $(100 \mu \mathrm{l}$ in culture medium) and including a no drug control were dispensed into 96-well plates, to which tightly synchronized (by alanine lysis) early ring-stage infected erythrocytes $(100 \mu \mathrm{l}$ at $2 \%$ haematocrit and 2\% parasitaemia in culture medium) were added. The plates were then placed in $3 \% \mathrm{O}_{2}, 7 \%$ $\mathrm{CO}_{2}, 90 \% \mathrm{~N}_{2}$ at $37^{\circ} \mathrm{C}$ for $22 \mathrm{~h}$ to mature (just prior to parasite release). Growth was halted and drug removed, by washing twice in ice-cold PBS. Erythrocytes were lysed by freezing at $-20^{\circ} \mathrm{C}$ and thawing. Aliquots of $0.5 \mathrm{mg} / \mathrm{ml}$ of $\mathrm{NBT}, 1 \mathrm{U} / \mathrm{ml}$ diaphorase and $50 \mu \mathrm{g} / \mathrm{ml}$ APAD in LDH buffer (100 mM Tris-HCl, pH 8.0, 50 mM Na L-lactate, 2.5\% $v / v$ Triton $\mathrm{X}-100)$ were added to the thawed cell pellets and incubated for $30 \mathrm{~min}$ in the dark at room temperature with shaking. The optical density (OD) at $655 \mathrm{~nm}$ of each well was measured in a BioRad 680 microplate reader. $\mathrm{OD}_{655}$ values were used to calculate growth by comparing the $\mathrm{OD}_{655}$ values at each drug concentration with that of the no drug control. To test the efficacy of artemisinin, artemether, artesunate and DHA, preparations of final concentrations of $0.1 \mathrm{nM}$ to $25 \mathrm{nM}$ were used, and for chloroquine and mefloquine, final concentrations of $1.25 \mathrm{nM}$ to $1 \mu \mathrm{M}$ were used.

\section{$\mathrm{IC}_{50}$ values and sequence alignments}

Dose-response data were modeled using a four-parameter fit and a variable slope, using Prism (Version 4.0a). Goodness of fit was assessed by $R^{2}$, and either noted in the text or highlighted if $<0.8$ in tables and figures. For the schizont maturation assay, the top parameter was constrained to $100 \%$ for assays using artemisinins but not for mefloquine or chloroquine. For the $p \mathrm{LDH}$ assay, the top and bottom parameters were constrained to 100 and 0 , respectively, for all drugs and, for the ${ }^{3} \mathrm{H}$ hypoxanthine incorporation assay, the top parameter was constrained to 100 . Data are summarized as a mean and 95\% CI for replicates of single parasite assays and a mean \pm SEM for results from multiple independent parasite assays.
The P. knowlesi and P. vivax orthologues of P. falciparum CRT, MDR1, and ATP6 (proteins associated with modulating sensitivities to chloroquine, mefloquine and artemisinins [13]), were aligned with P. falciparum 3D7 strain, as a drug sensitive control. $H$ strain and Sal-1 strain sequences were used for P. knowlesi and P. vivax, respectively. Wild-type and polymorphism sequence data were taken from PlasmoDB [14]. Alignments were performed in MacVector (version 11.0.2).

\section{Results}

Patient recruitment

Patient isolate data from those obtained in Sarikei and Sibu hospitals, between March and September 2010, are shown in Table 1. A total of nine patients were recruited into this study, six with $P$. knowlesi, two with $P$. vivax and one with $P$. falciparum monoinfections. Infections were initially diagnosed by microscopy and confirmed later by nested PCR $[2,6]$.

\section{Parasite development}

In vitro development data for each isolate used during growth assays are shown in Table 1. P. knowlesi field isolates were seeded into growth assays with a starting parasitaemia ranging from 0.4 to $7 \%$. The percentage of schizonts present at the start of the assays ranged from 6 to 33\%. Good in vitro development was demonstrated for all P. knowlesi field isolates, with 53 to $94 \%$ of asexual parasites reaching the schizont stage, over a development period of 12 to $17 \mathrm{~h}$. Development was similar for $P$. falciparum and $P$. vivax field isolates (Table 1).

\section{Growth assays: schizont maturation}

Dose-response curves were used to derive $\mathrm{IC}_{50}$ values, which are presented in Figure 1, together with mean values in Table 2. All the isolates from humans were sensitive to artemisinin and its derivatives, with $\mathrm{IC}_{50}$ values in the low $\mathrm{nM}$ range (from 0.38 to $6.8 \mathrm{nM}$ ). The $\mathrm{IC}_{50}$ values for chloroquine on $P$. knowlesi from humans were relatively high, with values ranging from 11 to $38 \mathrm{nM}$ but were similar to those derived for the single $P$. falciparum isolate (28 (20 to 38 ) nM; $\left.\mathrm{R}^{2}=0.93\right)$ and the two $P$. vivax isolates (33 (10 to 110$)$ and 18 (11 to 27$) n M ; R^{2}=0.45$ and 0.95 , respectively) that were studied.

Plasmodium knowlesi isolates were least sensitive to mefloquine, with a mean $\mathrm{IC}_{50}$ value of $26 \mathrm{nM}$ (Table 2). The $\mathrm{IC}_{50}$ value for mefloquine on the single $P$. falciparum field isolate (6.3 (2.5 to 16) $\mathrm{nM} ; \mathrm{R}^{2}=0.97$ ) was four-fold less than that of the average $P$. knowlesi $\mathrm{IC}_{50}$ value and 2.5 -fold less than the lowest $P$. knowlesi $\mathrm{IC}_{50}$ value (16 (12 to 22$\left.) n M ; R^{2}=0.96\right)$. 


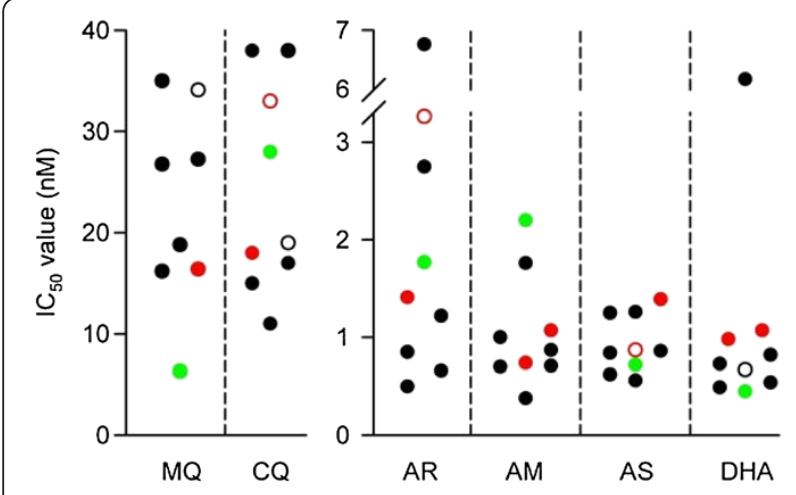

Figure $1 \mathrm{IC}_{50}$ values for anti-malarial drugs against Plasmodium isolates, using the schizont maturation assay. Data points represent mean $I_{50}$ values $(n M)$ derived from single experiments performed in triplicate. Plasmodium knowlesi (black symbols), P. vivax (red symbols) and $P$. falciparum (green symbols). Filled symbols indicate $R^{2}$ values $\geq 0.8$, with unfilled symbols indicting $R^{2}$ values $<0.8$. Mefloquine, MQ; Chloroquine, CQ; Artemisinin, AR; Artemether, AM; Artesunate, AS; Dihydroartemisinin, DHA. Note that there is only mefloquine data for one of the two P. vivax isolates.

\section{Growth assays: $p \mathrm{LDH}$}

To validate the results of the drug assays on $P$. knowlesi from patients, anti-malarials were assessed against the well characterized $P$. knowlesi $\mathrm{H}$ strain grown in vitro in rhesus erythrocytes. To determine whether data generated by the $p \mathrm{LDH}$ method (as used to study the $\mathrm{H}$ strain) would be comparable with the adapted schizont maturation method (as used in the field study) a parallel study was performed, using artesunate. The $\mathrm{IC}_{50}$ value for artesunate against the $\mathrm{H}$ strain was $2.0(0.93$ to 4.2$) \mathrm{nM}$ $\left(\mathrm{R}^{2}=0.39\right)$, using the $p \mathrm{LDH}$ assay (from a single experiment repeated in quintuplicate), and 1.2 (0.88 to 1.6$) \mathrm{nM}$ $\left(R^{2}=0.95\right)$, using the schizont maturation assay (from a single experiment repeated in triplicate), demonstrating that the two assay methods are comparable, at least in the

Table $2 P$. knowlesi drug sensitivity data

\begin{tabular}{lll}
\hline Drug & $\begin{array}{l}\mathrm{IC}_{50} \text { values }(\mathrm{nM}) \text { for } \\
\boldsymbol{P} \text {. knowlesi isolates } \\
\text { (maturation assay) }^{\mathbf{a}}\end{array}$ & $\begin{array}{l}\mathrm{IC}_{50} \text { values }(\mathrm{nM}) \text { for } \\
\boldsymbol{P} \text {. knowlesi } \mathrm{H} \text { strain } \\
(\boldsymbol{p} \text { LDH assay) }\end{array}$ \\
\hline Mefloquine & $26( \pm 3.1)$ & $25(7.4 \text { to } 81)^{\mathrm{c}}$ \\
Chloroquine & $23( \pm 4.8)$ & $3.2(2.2$ to 4.7$)$ \\
Artemisinin & $2.1( \pm 0.99)$ & $0.80(0.35 \text { to } 1.9)^{\mathrm{c}}$ \\
Artemether & $0.90( \pm 0.19)$ & $0.84(0.34 \text { to } 2.1)^{\mathrm{c}}$ \\
Artesunate & $0.90( \pm 0.12)$ & $2.0(0.93 \text { to } 4.2)^{\mathrm{c}}$ \\
DHA & $1.6( \pm 0.92)$ & $0.79(0.62$ to 1.0$)$
\end{tabular}

${ }^{\mathrm{a}}$ Mean $( \pm$ SEM), from 6 independent experiments (each of which was performed in triplicate).

${ }^{\mathrm{b}}$ Mean $(95 \% \mathrm{Cls})$, from a single experiment repeated in quintuplicate. ${ }^{\mathrm{c}} \mathrm{R}^{2}<0.8$.

$\mathrm{IC}_{50}$ values are presented for six anti-malarials on $P$. knowlesi field isolates and laboratory $\mathrm{H}$ strain. case of artemisinins. Further $\mathrm{IC}_{50}$ values derived from $p \mathrm{LDH}$ assays are presented in Table 2 . All $\mathrm{IC}_{50}$ values for field isolates and the $P$. knowlesi $\mathrm{H}$ strain for artemisinins are highly comparable.

For mefloquine, the $\mathrm{IC}_{50}$ value against the laboratory $\mathrm{H}$ strain (25 (7.4 to 81$\left.) \mathrm{nM} ; \mathrm{R}^{2}=0.40\right)$ was nearly identical to the mean $\mathrm{IC}_{50}$ value of human isolates $(26 \mathrm{nM})$. The $\mathrm{IC}_{50}$ values suggest that $P$. knowlesi may be intrinsically insensitive to mefloquine. To ensure that the mefloquine stock used in field studies had not degraded during the study, an aliquot was tested after shipping it back to St. George's, University of London after the study. Using hypoxanthine incorporation as a measure of growth [15] in a single assay (performed in quadruplicate) with $P$. falciparum (3D7) parasites cultured in vitro in human erythrocytes, the $\mathrm{IC}_{50}$ value was 9.7 (5.5 to 17$) \mathrm{nM}\left(\mathrm{R}^{2}=0.89\right)$, confirming that potency of mefloquine was maintained.

Interestingly, the $\mathrm{H}$ strain was over 7 -fold more sensitive to chloroquine than the average value for field isolates, having an $\mathrm{IC}_{50}$ value of $3.2(2.2$ to 4.7$) \mathrm{nM}\left(\mathrm{R}^{2}=0.86\right)$. This value was also well below the lowest calculated $\mathrm{IC}_{50}$ value (11 (5.3 to 24) $\mathrm{nM} ; \mathrm{R}^{2}=0.94$ ) for a field isolate.

\section{Sequence alignments}

Point mutations associated with change in the sensitivity of $P$. falciparum to chloroquine, mefloquine and the artemisinins [13] were analysed, and similarities and differences in sequences encoded by $P$. knowlesi and $P$. vivax homologues are reported in Figure 2. Known loci at which mutations can reduce drug sensitivity were highly conserved between $P$. knowlesi $\mathrm{H}, P$. vivax Sal-1 MDR1 homologues and MDR1 in drug sensitive P. falciparum 3D7. PfATP6 orthologues demonstrated polymorphism in amino acids in about two thirds of the

\begin{tabular}{|c|c|c|c|c|c|c|c|c|}
\hline \multicolumn{3}{|c|}{ PfCRT } & \multicolumn{3}{|c|}{ PfATP6 } & \multicolumn{3}{|c|}{$P$ FMDR 1} \\
\hline$P f$ & $P k$ & $P_{V}$ & $P f$ & $P k$ & $P V$ & $P f$ & $P k$ & $P V$ \\
\hline C72S & $71 \mathrm{C}$ & $71 \mathrm{C}$ & I89T & $89 \mathrm{~V}$ & $89 \mathrm{~V}$ & N86Y & $91 \mathrm{~N}$ & $91 \mathrm{~N}$ \\
\hline M74I & $73 M$ & $73 M$ & H243Y & $243 \mathrm{H}$ & $243 \mathrm{H}$ & $\mathrm{Y} 184 \mathrm{~F}$ & $189 \mathbf{Y}$ & $189 \mathrm{Y}$ \\
\hline N75E & $74 \mathbf{E}$ & $74 \mathrm{E}$ & L263E & $263 \mathrm{~S}$ & $263 A$ & N1042D & $1081 \mathrm{~N}$ & $1082 \mathrm{~N}$ \\
\hline K76T & $75 \mathrm{~K}$ & $75 \mathbf{K}$ & $2402 \mathrm{~V}$ & $402 \mathrm{~A}$ & $402 \mathrm{~A}$ & D1246Y & 1293D & 1292D \\
\hline H97Q & 96H & 96H & E431K & $429 T$ & $429 \mathrm{~A}$ & & & \\
\hline S219A & $218 \mathrm{~A}$ & $218 \mathrm{~A}$ & E432K & $430 \mathrm{~S}$ & $430 \mathrm{~s}$ & & & \\
\hline A220S & $219 \mathrm{~S}$ & $219 \mathrm{~S}$ & Q574P & - & - & & & \\
\hline $\mathrm{Q} 271 \mathrm{E}$ & 2700 & 2700 & A623E & - & - & & & \\
\hline $\mathrm{N} 326 \mathrm{~S} / \mathrm{D}$ & $325 \mathrm{~N}$ & $325 \mathrm{~N}$ & S769N & $708 \mathrm{~K}$ & $718 \mathrm{~K}$ & & & \\
\hline I356T/L & $355 I$ & $355 \mathrm{I}$ & & & & & & \\
\hline R371I & $370 \mathrm{M}$ & $370 \mathrm{M}$ & & & & & & \\
\hline \multicolumn{9}{|c|}{$\begin{array}{l}\text { Figure } 2 \text { Alignment of CRT, ATP6, and MDR1 homologues of } \\
P \text {. knowlesi } \mathrm{H} \text { strain and } P \text {. vivax Sal-1 strain, against } P \text {. falciparum } \\
\text { 3D7 and strains with known point mutations associated with drug } \\
\text { resistance. Amino acids are colour coded, with green representing } \\
\text { the drug sensitive } P \text {. falciparum 3D7, and red representing amino } \\
\text { acid changes found in } P \text {. falciparum isolates that have a change in } \\
\text { drug sensitivity. }\end{array}$} \\
\hline
\end{tabular}


residues previously examined for effects on drug sensitivity, including L263 [16-18].

The CRT orthologues were fairly well conserved with the exceptions of S219A, A220S, and R371M in the chloroquine sensitive strain. These differences do not confer chloroquine resistance to the $P$. knowlesi $\mathrm{H}$ strain. Agreement in sequences in these polymorphic regions was higher between $P$. knowlesi $\mathrm{H}$ and $P$. vivax Sal-1 than between either strain and P. falciparum 3D7 consistent with a closer phylogenetic relationship between the former species [19].

\section{Discussion}

In vitro culture of $P$. knowlesi has only recently been achieved in human erythrocytes [20,21]. However, drug sensitivity assays of natural human infections can only be assessed in short term cultures. These allowed the successful application of drug sensitivity micro-assays such as the WHO Mark III micro-assay test after adaptation to the quotidian life cycle of the parasite and frequent (every 2 to $2.5 \mathrm{~h}$ ) monitoring of development to allow assessment of when most parasites were mature schizonts in control samples.

The excellent in vitro efficacy of the artemisinins against both human $P$. knowlesi (mean $\mathrm{IC}_{50}$ values $<2.2 \mathrm{nM}$ ) and the laboratory $(\mathrm{H})$ strain is consistent with recent observations made on patients treated with artesunate, where no mortality was observed after treatment [22]. These results also agree with data from animal models, which demonstrate the successful clearance of $P$. knowlesi parasites from infected rhesus monkeys, by artemisinin in combination therapy with naphthoquine [23]. In addition, William et al. [24] in a retrospective analysis of clinical cases of knowlesi malaria in Sabah, reported the successful treatment of patients by artemether-lumefantrine combination therapy. This study also noted that where knowlesi malaria developed signs of severity, intravenous artesunate was effective [24].

Intriguingly, chloroquine $\mathrm{IC}_{50}$ values from this field study, including those derived against $P$. knowlesi (with values ranging from 11 to $38 \mathrm{nM}$ ), were higher than that of the laboratory P. knowlesi $\mathrm{H}$ strain $(3.2 \mathrm{nM})$, although the reason for this is unclear. In general, the values are comparable with chloroquine-sensitive laboratory strains of $P$. falciparum, having $\mathrm{IC}_{50}$ values ranging from 8 to $15 \mathrm{nM}$ [25-27]. These values all fall below the $100 \mathrm{nM}$ threshold used to define chloroquine resistance $[27,28]$ and there is no evidence for clinical chloroquine resistance reported in P. knowlesi. Chloroquine is effective both as a monotherapy and when used in combination with primaquine $[1,24,29]$. Consistent with these findings, Tyagi et al. [30] have recently reported that CRT (and DHFR) sequences from P. knowlesi clinical isolates collected in the Andaman and Nicobar Islands, India, were all found to be wild-type (with close homology to the CRT sequence of chloroquine sensitive P. falciparum parasites - see Figure 2 and below). Nevertheless, continued monitoring of chloroquine sensitivity in $P$. knowlesi might be prudent bearing in mind the history of chloroquine resistance development in other malarial species.

Evidence suggests that transmission of $P$. knowlesi to humans in Sarawak remains zoonotic and, thus, ostensibly free from mefloquine drug selection pressure. However, results with mefloquine consistently showed a low sensitivity in $P$. knowlesi field isolates, when compared with that of $P$. falciparum. The mean $\mathrm{IC}_{50}$ value for mefloquine calculated for the 6 P. knowlesi isolates is $26 \mathrm{nM}$, which is just above the value used to define mefloquine resistance in P. falciparum $(>24 \mathrm{nM})$ in some reports $[31,32]$ but well below that reported by others $(>119 \mathrm{nM})$ [33]. Importantly, the reduced $P$. knowlesi response in human isolates was also observed in the P. knowlesi $\mathrm{H}$ experimental line. Given the efficacy of the mefloquine used was confirmed after the end of the study, these results indicate an innate tolerance of $P$. knowlesi to mefloquine. These findings also suggest the strong possibility of treatment failure if mefloquine is used as mono or combination therapy for $P$. knowlesi and is supported by reports of mefloquine treatment failure in rhesus monkeys infected with P. knowlesi [34], as well as recent cases of mefloquine treatment failure in humans with knowlesi malaria [35]. On this basis, mefloquine should be used with caution for the treatment of knowlesi malaria, or indeed for prophylaxis against malaria in areas where acquiring knowlesi is a risk until larger studies have been undertaken.

There is reasonable conservation of MDR1 sequences between $P$. vivax Sal-1 and P. knowlesi $\mathrm{H}$ and the P. falciparum 3D7 reference strain. Previous studies on P. falciparum found that increased $m d r 1$ copy number conferred a mefloquine resistant phenotype [36] and risk of treatment failure, although $P$. knowlesi $m d r 1$ copy number in the isolates reported in the current study have not been determined. Alignments of the P. vivax Sal-1 and P. knowlesi $\mathrm{H}$ CRT and ATP6 orthologues with the P. falciparum 3D7 sequence revealed several polymorphic differences between the $P$. vivax and P. knowlesi alignments and that of the P. falciparum. These substitutions do not alter sensitivity of $P$. knowlesi to artemisinins and give insights into the possible contributions of these residues to artemisinin sensitivity in P. falciparum.

Here, it has been established that it is possible to culture in vitro P. knowlesi in human erythrocytes in the short term, when taken ex vivo. In addition, the successful adaption of the schizont development assay to determine anti-malarial drug sensitivities of $P$. knowlesi field isolates has been shown. Using this adapted method, this study has demonstrated that chloroquine and artemisinin 
based drugs are effective against $P$. knowlesi parasites. Conversely this study has shown poor sensitivity of $P$. knowlesi field isolates and laboratory $\mathrm{H}$ strain to mefloquine, suggesting innate reduced sensitivity of the parasite to this important anti-malarial drug.

\section{Competing interests}

The authors declare that they have no competing interests.

\section{Authors' contributions}

The study was conceived by SK and designed by HMS, CHMK, BS, JCS and SK. The assays were performed by FAF with support from HMS, AS, MAA, LCW, JCS and EMP. The manuscript was prepared by FAF, HMS, and SK. All authors had the opportunity to read and approve the manuscript.

\section{Acknowledgements}

We gratefully acknowledge the staff at Hospital Sarikei especially Mr Wong Ching Toh, Mr Pek Peng Chin, Mdm Siti Syartinah and Mdm Raymand Johan for helping with patient recruitment and Mdm Dayang Shuaisah Awang Mohamad and the staff at the Malaria Research Centre at UNIMAS. Finally, we would like to thank the patients who so kindly agreed to be a part of this study, and without whom this research would not have been possible.

\section{Funding}

FAF was funded by the MRC-Doctoral Training Grant G0800110. This study was funded by the Medical Research Council (MRC) UK; Grant number G0801971 and the European Community's Seventh Framework Programme (FP7/2007-2013), EVIMALAR network of Excellence under grant agreement $\mathrm{N}^{\circ}$ 242095 and NANOMAL under grant agreement $N^{\circ} 304948$.

\section{Author details}

'Division of Clinical Sciences, Centre for Infection and Immunity, St. George's, University of London, London SW17 ORE, UK. ${ }^{2}$ Malaria Research Centre, University Malaysia Sarawak, Kuching 93150, Malaysia. ${ }^{3}$ Pathology Laboratory, Hospital Sarikei, Sarikei 96100, Malaysia. ${ }^{4}$ Biomedical Primate Research Centre, Lange Kleiweg 161, GJ Rijswijk, The Netherlands. ${ }^{5}$ School of Medicine, University of St Andrews, Medical and Biological Sciences Building, North Haugh, St Andrews KY16 9TF, UK.

Received: 18 September 2013 Accepted: 12 November 2013 Published: 19 November 2013

\section{References}

1. Singh B, Daneshvar C: Human infections and detection of Plasmodium knowlesi. Clin Microbiol Rev 2013, 26:165-184.

2. Singh B, Kim Sung L, Matusop A, Radhakrishnan A, Shamsul SS, Cox-Singh J, Thomas A, Conway DJ: A large focus of naturally acquired Plasmodium knowlesi infections in human beings. Lancet 2004, 363:1017-1024.

3. Vythilingam I, Tan CH, Asmad M, Chan ST, Lee KS, Singh B: Natural transmission of Plasmodium knowlesi to humans by Anopheles latens in Sarawak, Malaysia. Trans R Soc Trop Med Hyg 2006, 100:1087-1088.

4. Cox-Singh J, Davis TM, Lee KS, Shamsul SS, Matusop A, Ratnam S, Rahman HA, Conway DJ, Singh B: Plasmodium knowlesi malaria in humans is widely distributed and potentially life threatening. Clin Infect Dis 2008, 46:165-171.

5. William T, Rahman HA, Jelip J, Ibrahim MY, Menon J, Grigg MJ, Yeo TW, Anstey NM, Barber BE: Increasing incidence of Plasmodium knowlesi malaria following control of $P$. falciparum and $P$. vivax malaria in Sabah, Malaysia. PLoS Negl Trop Dis 2013, 7:e2026.

6. Lee KS, Divis PC, Zakaria SK, Matusop A, Julin RA, Conway DJ, Cox-Singh J, Singh B: Plasmodium knowlesi: reservoir hosts and tracking the emergence in humans and macaques. PLoS Pathog 2011, 7:e1002015.

7. WHO Mark II assay. www.who.int/malaria/publications/atoz/markiii.pdf.

8. Srinivas SD, Puri SK: Time course of in vitro maturation of intraerythrocytic malaria parasite: a comparison between Plasmodium falciparum and Plasmodium knowlesi. Mem Inst Oswaldo Cruz 2002, 97:901-903.

9. Wernsdorfer $\mathrm{WH}$, Tasanor $\mathrm{O}$, Wernsdorfer $\mathrm{G}:$ In vitro drug sensitivity testing in Plasmodium vivax. Wien Klin Wochenschr 2008, 120:30-33.
10. Howard RJ, Barnwell JW, Kao V: Antigenic variation of Plasmodium knowlesi malaria: identification of the variant antigen on infected erythrocytes. Proc Natl Acad Sci U S A 1983, 80:4129-4133.

11. Kocken $\mathrm{CH}$, Ozwara $\mathrm{H}$, van der Wel A, Beetsma AL, Mwenda JM, Thomas AW Plasmodium knowlesi provides a rapid in vitro and in vivo transfection system that enables double-crossover gene knockout studies. Infect Immun 2002, 70:655-660.

12. Druilhe $\mathrm{P}$, Moreno A, Blanc C, Brasseur PH, Jacquier P: A colorimetric in vitro drug sensitivity assay for Plasmodium falciparum based on a highly sensitive double-site lactate dehydrogenase antigen-capture enzyme-linked immunosorbent assay. Am J Trop Med Hyg 2001, 64:233-241.

13. Ecker A, Lehane AM, Fiddock DA: Molecular markers of Plasmodium resistance to antimalarials. In Treatment and Prevention of Malaria: Antimalarial Drug Chemistry, Action and Use. Edited by Staines HM, Krishna S. Basel: Springer Basel; 2012:249-280.

14. PlasmoDB. www.plasmodb.org/plasmo/.

15. Desjardins RE, Canfield CJ, Haynes JD, Chulay JD: Quantitative assessment of antimalarial activity in vitro by a semiautomated microdilution technique. Antimicrob Agents Chemother 1979, 16:710-718.

16. Pulcini S, Staines HM, Pittman JK, Slavic K, Doerig C, Halbert J, Tewari R, Shah F, Avery MA, Haynes RK, Krishna S: Expression in yeast links field polymorphisms in PfATP6 to in vitro artemisinin resistance and identifies new inhibitor classes. J Infect Dis 2013. in press.

17. Uhlemann AC, Cameron A, Eckstein-Ludwig U, Fischbarg J, Iserovich $P$, Zuniga FA, East M, Lee A, Brady L, Haynes RK, Krishna S: A single amino acid residue can determine the sensitivity of SERCAs to artemisinins. Nat Struct Mol Biol 2005, 12:628-629.

18. Valderramos SG, Scanfeld D, Uhlemann AC, Fidock DA, Krishna S: Investigations into the role of the Plasmodium falciparum SERCA (PfATP6) L263E mutation in artemisinin action and resistance. Antimicrob Agents Chemother 2010, 54:3842-3852.

19. Tachibana S, Sullivan SA, Kawai S, Nakamura S, Kim HR, Goto N, Arisue N, Palacpac NM, Honma H, Yagi M, Tougan T, Katakai Y, Kaneko O, Mita T, Kita K, Yasutomi Y, Sutton PL, Shakhbatyan R, Horii T, Yasunaga T, Barnwell JW, Escalante AA, Carlton JM, Tanabe K: Plasmodium cynomolgi genome sequences provide insight into Plasmodium vivax and the monkey malaria clade. Nat Genet 2012, 44:1051-1055.

20. Lim C, Hansen E, DeSimone TM, Moreno Y, Junker K, Bei A, Brugnara C, Buckee CO, Duraisingh MT: Expansion of host cellular niche can drive adaptation of a zoonotic malaria parasite to humans. Nat Commun 2013, 4:1638.

21. Moon RW, Hall J, Rangkuti F, Ho YS, Almond N, Mitchell GH, Pain A, Holder AA, Blackman MJ: Adaptation of the genetically tractable malaria pathogen Plasmodium knowlesi to continuous culture in human erythrocytes. Proc Natl Acad Sci U S A 2013, 110:531-536.

22. Barber BE, William T, Grigg MJ, Menon J, Auburn S, Marfurt J, Anstey NM, Yeo TW: A prospective comparative study of knowlesi, falciparum, and vivax malaria in sabah, malaysia: high proportion with severe disease from Plasmodium knowlesi and Plasmodium vivax but no mortality with early referral and artesunate therapy. Clin Infect Dis 2013, 56:383-397.

23. Wang JY, Ding DB, Li GF, Zhao JH: Therapeutic efficacy of naphthoquine phosphate combined with artemisinine against Plasmodium knowlesi. Zhongguo Ji Sheng Chong Xue Yu Ji Sheng Chong Bing Za Zhi 2008, 26:442-444.

24. William T, Menon J, Rajahram G, Chan L, Ma G, Donaldson S, Khoo S, Frederick C, Jelip J, Anstey NM, Yeo TW: Severe Plasmodium knowlesi malaria in a tertiary care hospital, Sabah, Malaysia. Emerg Infect Dis 2011 17:1248-1255.

25. Co EM, Dennull RA, Reinbold DD, Waters NC, Johnson JD: Assessment of malaria in vitro drug combination screening and mixed-strain infections using the malaria Sybr green I-based fluorescence assay. Antimicrob Agents Chemother 2009, 53:2557-2563.

26. Tamez PA, Lantvit D, Lim E, Pezzuto JM: Chemosensitizing action of cepharanthine against drug-resistant human malaria, Plasmodium falciparum. J Ethnopharmacol 2005, 98:137-142.

27. Zalis MG, Pang L, Silveira MS, Milhous WK, Wirth DF: Characterization of Plasmodium falciparum isolated from the Amazon region of Brazil: evidence for quinine resistance. Am J Trop Med Hyg 1998, 58:630-637.

28. Basco LK: Molecular epidemiology of malaria in cameroon. XX. Experimental studies on various factors of in vitro drug sensitivity assays 
using fresh isolates of Plasmodium falciparum. Am J Trop Med Hyg 2004, 70:474-480.

29. Daneshvar C, Davis TM, Cox-Singh J, Rafa'ee MZ, Zakaria SK, Divis PC, Singh B: Clinical and parasitological response to oral chloroquine and primaquine in uncomplicated human Plasmodium knowlesi infections. Malar J 2010, 9:238.

30. Tyagi RK, Das MK, Singh SS, Sharma YD: Discordance in drug resistanceassociated mutation patterns in marker genes of Plasmodium falciparum and Plasmodium knowlesi during coinfections. J Antimicrob Chemother 2013, 68:1081-1088.

31. Cerutti Junior C, Marques C, Alencar FE, Durlacher RR, Alween A, Segurado AA, Pang LW, Zalis MG: Antimalarial drug susceptibility testing of Plasmodium falciparum in Brazil using a radioisotope method. Mem Inst Oswaldo Cruz 1999, 94:803-809.

32. Pickard AL, Wongsrichanalai C, Purfield A, Kamwendo D, Emery K, Zalewski C, Kawamoto F, Miller RS, Meshnick SR: Resistance to antimalarials in Southeast Asia and genetic polymorphisms in pfmdr1. Antimicrob Agents Chemother 2003, 47:2418-2423.

33. Price RN, Cassar C, Brockman A, Duraisingh M, van Vugt M, White NJ, Nosten F, Krishna S: The pfmdr1 gene is associated with a multidrugresistant phenotype in Plasmodium falciparum from the western border of Thailand. Antimicrob Agents Chemother 1999, 43:2943-2949.

34. Tripathi R, Awasthi A, Dutta GP: Mefloquine resistance reversal action of ketoconazole - a cytochrome P450 inhibitor, against mefloquineresistant malaria. Parasitology 2005, 130:475-479.

35. Lau YL, Tan LH, Chin LC, Fong MY, Noraishah MA, Rohela M: Plasmodium knowlesi reinfection in human. Emerg Infect Dis 2011, 17:1314-1315.

36. Price RN, Uhlemann AC, Brockman A, McGready R, Ashley E, Phaipun L, Patel R, Laing K, Looareesuwan S, White NJ, Nosten F, Krishna S: Mefloquine resistance in Plasmodium falciparum and increased pfmdr1 gene copy number. Lancet 2004, 364:438-447.

doi:10.1186/1475-2875-12-425

Cite this article as: Fatih et al: Susceptibility of human Plasmodium

knowlesi infections to anti-malarials. Malaria Journal 2013 12:425.

\section{Submit your next manuscript to BioMed Central and take full advantage of:}

- Convenient online submission

- Thorough peer review

- No space constraints or color figure charges

- Immediate publication on acceptance

- Inclusion in PubMed, CAS, Scopus and Google Scholar

- Research which is freely available for redistribution 